\title{
Effects of the transition time between muscle-tendon stretch and shortening on mechanical efficiency
}

Accepted: 13 December 2005/Published online: 17 January 2006 (C) Springer-Verlag 2006

\begin{abstract}
The net mechanical efficiency of positive work $\left(\eta_{\text {pos }}\right)$ has been shown to increase if it is immediately preceded by negative work. This phenomenon is explained by the storage of elastic energy during the negative phase and its release during the subsequent positive phase. If a transition time $(T)$ takes place, the elastic energy is dissipated into heat. The aim of the present study was to investigate the relationship between $\eta_{\text {pos }}$ and $T$, and to determine the minimal $T$ required so that $\eta_{\text {pos }}$ reached its minimal value. Seven healthy male subjects were tested during four series of loweringraising of the body mass. In the first series $\left(S_{0}\right)$, the negative and positive phases were executed without any transition time. In the three other series, $T$ was varied by a timer $\left(0.12,0.24\right.$ and $0.56 \mathrm{~s}$ for series $S_{1}, S_{2}$ and $S_{3}$, respectively). These exercises were performed on a force platform sensitive to vertical forces to measure the mechanical work and a gas analyser was used to determine the energy expenditure. The results indicated that $\eta_{\text {pos }}$ was the highest $(31.1 \%)$ for the series without any transition time $\left(S_{0}\right)$. The efficiencies observed with transition times $\left(S_{1}, S_{2}\right.$ and $\left.S_{3}\right)$ were $27.7,26.0$ and $23.8 \%$, respectively, demonstrating that $T$ plays an important role for mechanical efficiency. The investigation of the relationship between $\eta_{\text {pos }}$ and $T$ revealed that the minimal $T$ required so that $\eta_{\text {pos }}$ reached its minimal value is $0.59 \mathrm{~s}$.
\end{abstract}

Y. Henchoz $(\bowtie) \cdot D$. Malatesta $\cdot$ A. Belli

Institut des Sciences du Sport et de l'Education Physique, Université de Lausanne, Bâtiment Provence,

1015 Lausanne, Switzerland

E-mail: yves.henchoz@unil.ch

Tel.: + 41-21-6923298

Fax: + 41-21-6923293

G. Gremion

University Hospital for Orthopaedic Surgery,

Lausanne, Switzerland
Keywords Mechanical efficiency · Elastic energy ·

Transition time $\cdot$ Stretch-shortening cycle

\section{Introduction}

The stretch-shortening cycle (SSC) is recognised as being responsible for an increase in mechanical efficiency while running (Asmussen and Bonde-Petersen 1974; Cavagna et al. 1964), squatting (Asmussen and Bonde-Petersen 1974; Thys et al. 1972) and jumping (Bosco et al. 1982; Thys et al. 1975). This enhancement is attributed to the possibility of reusing in the positive work phase of exercise the elastic energy stored during the negative work phase in the series elastic elements and, to a lesser extent, in the parallel elastic elements (Cavagna et al. 1985), improving the mechanical efficiency of positive work $\left(\eta_{\text {pos }}\right)$. Evidence for this was given by Thys et al. (1972) who measured a higher mechanical efficiency for lowering-raising of the body mass in a rebound condition than in a no-rebound condition for which the muscles were relaxed between the flexion (lowering) and the extension (raising). In the same study, the time delay, or transition time $(T)$, between the end of the flexion and the beginning of the extension was approximately equal to $0 \mathrm{~s}$ for the rebound condition and $0.6 \mathrm{~s}$ for the norebound condition. It was concluded that the stored elastic energy is turned into heat if the muscle is allowed to relax.

A second parameter of the elastic energy loss might be the duration of $T$, the percentage of released elastic energy being maximum when $T=0 \mathrm{~s}$ and progressively decreasing until it reaches its minimal value for a certain $T$ value. However, to our knowledge, mechanical efficiency has not been measured in a study including several $T$ values. Consequently, the relationship between mechanical efficiency and $T$ is unknown.

The purpose of the present study, therefore, was to investigate the relationship between $\eta_{\text {pos }}$ and $T$, and to determine the minimal $T$ required so that $\eta_{\text {pos }}$ reached its minimal value. 


\section{Methods}

\section{Subjects}

Seven physical education students took part in this experiment after giving their written informed consent. They were [mean (SD)] $24.4(1.0)$ years old, $1.78(0.07) \mathrm{m}$ tall, with a body mass of $70.6(10.9) \mathrm{kg}$. The study was conducted according to the 1964 declaration of Helsinki.

\section{Experimental protocol}

All subjects performed four series of flexion-extensions of the lower limbs at a frequency of 20 cycles $\min ^{-1}$. Each series was 4 min long. The four series were randomly executed with 5-min breaks and differed only in the duration of $T$, as dictated by a timer. The test apparatus involved rhythm recordings of two beeps that repeat every $3 \mathrm{~s}$. At the first beep, the subjects executed a flexion on a vertical adjustable seat. They stayed in an isometric squatting position $\left(\sim 90^{\circ}\right.$ angle of the knee joints), just in contact with the seat so that the extensor muscles of the lower limbs remained contracted, until the second beep at which they performed an extension. The two beeps were spaced with $0.75,1$ and $1.25 \mathrm{~s}$ for series $S_{1}, S_{2}$ and $S_{3}$, respectively. In series $S_{0}$, only one beep was sounded, at which time the subjects performed a flexion immediately followed by an extension. Flexions and extensions were executed as fast as possible so that the stretching and shortening velocities would be approximately constant in the four series.

\section{Apparatus}

The four series were performed on a force platform (Kistler, type 9290) sensitive to vertical forces. Data were sampled at a frequency of $500 \mathrm{~Hz}$ and recorded on a personal computer. Oxygen consumption $\left(\dot{V} \mathrm{O}_{2}\right)$ and carbon dioxide production $\left(\dot{V} \mathrm{CO}_{2}\right)$ were measured breath-by-breath with a gas analyser (CPX, MedGraphics $\left({ }^{\circledR}\right)$. Metabolic data were averaged over $20 \mathrm{~s}$ intervals. Only the last minute of all 4-min-long measurements was taken into account for the results. The 3-min delay was considered as sufficient so that the subjects reached a steady state (Whipp and Wasserman 1972).

\section{Data analysis}

In series $S_{1}, S_{2}$ and $S_{3}$, the intervals between the two beeps of the timer include both the flexion and transition time phases and, thus, cannot be considered as an exact measurement of $T$. Before the experiment, the flexion phase was measured to last approximately $0.5 \mathrm{~s}$.
Therefore, the $T$ values were expected to be $0.25,0.5$ and $0.75 \mathrm{~s}$ for series $S_{1}, S_{2}$ and $S_{3}$, respectively. An objective measure of $T$ was done on a personal computer by detecting, for each flexion-extension cycle, the time during which the subjects were static. In order to take the vertical oscillations of the subjects during the transition time into account, the subjects were considered to be static when their vertical speed was between -0.1 and $0.1 \mathrm{~m} \mathrm{~s}^{-1}$. For each cycle, flexion and extension times were also measured, and used to calculate the average velocities of flexion $\left(v_{\mathrm{ff}}\right)$ and extension $\left(v_{\mathrm{ex}}\right)$.

\section{Metabolic analysis}

A table of correspondence gives the energetic equivalent of $\mathrm{O}_{2}\left(\mathrm{EEO}_{2}\right)$ consumption for each value of respiratory quotient. Net metabolic power $\left(\dot{E}_{\text {meta }}\right)$ associated with the exercise was calculated as follows:

$\dot{E}_{\text {meta }}(\mathrm{W})=\left(\dot{V} \mathrm{O}_{2}-\dot{V} \mathrm{O}_{2 \text { rest }}\right) \mathrm{EEO}_{2}$

Since the time of measurement was equal to $1 \mathrm{~min}$, the corresponding metabolic energy expenditure $\left(E_{\text {meta }}\right)$ was equal to the metabolic power value $\left(\dot{E}_{\text {meta }}\right)$.

\section{Mechanical analysis}

The vertical velocity of the centre of body mass $\left(v_{\mathrm{v}}\right)$ is the integral of vertical acceleration $\left(a_{\mathrm{v}}\right)$ and was obtained directly by electronically integrating the signal measured by the force platform $\left(F_{\mathrm{v}}\right)$ :

$F_{\mathrm{v}}-m \times g=m \times a_{\mathrm{v}}$

where $m$ is the body mass and $g$ is the acceleration due to gravity $\left(9.81 \mathrm{~m} \mathrm{~s}^{-2}\right)$. The quantity $F_{\mathrm{v}}-m \times g$ was measured directly during each exercise by zeroing the force platform immediately before each series.

The vertical velocity of the centre of body mass $\left(v_{\mathrm{v}}\right)$ was then determined by:

$v_{\mathrm{v}}\left(\mathrm{m} \mathrm{s}^{-1}\right)=\int a_{\mathrm{v}} \mathrm{d} t=\int\left(\frac{F_{\mathrm{v}}-m \times g}{m}\right) \mathrm{d} t+v_{0}$

where $v_{0}$ is the integration constant and equals zero when the subject begins to move from the erect position.

The vertical displacement of centre of body mass $\left(h_{\mathrm{v}}\right)$ was calculated by a successive integration of $v_{\mathrm{v}}$ :

$h_{\mathrm{v}}(\mathrm{m})=\int v_{\mathrm{v}} \mathrm{d} t+h_{0}$

where $h_{0}$ is the integration constant and corresponds to the vertical position of the subjects in the erect position (vertical zero position).

The external mechanical work $\left(W_{\text {ext }}\right)$ was the sum of the positive and negative work produced during the time of measurement (1 $\mathrm{min})$ and was obtained as follows: 
$W_{\text {ext }}(\mathbf{J})=\int_{t=0}^{t=60} F_{\mathrm{v}} v_{\mathrm{v}} \mathrm{d} t$

Positive and negative work were calculated separately. The work performed was considered to be positive $\left(W_{\text {pos }}\right)$ when the subject was going up $\left(v_{\mathrm{v}}>0\right)$ and negative $\left(W_{\text {neg }}\right)$ when the subject was going down $\left(v_{\mathrm{v}}<0\right)$. Horizontal accelerations (forward and lateral) and internal work, such as that due to viscosity of the body tissues or to contraction of the muscles not leading to a vertical displacement, were neglected (Thys et al. 1972).

Net efficiency $\left(\eta_{\text {net }}\right)$ was calculated as the ratio of $W_{\text {ext }}$ to $E_{\text {meta }}$ (Gaesser and Brooks 1975; Stainbsy et al. 1980): $\eta_{\text {net }}=W_{\text {ext }} E_{\text {meta }}^{-1}=\left(W_{\text {pos }}+W_{\text {neg }}\right) E_{\text {meta }}^{-1}$.

$W_{\text {pos }}$ includes a certain amount of mechanical work done by the contractile component of the muscles and also a proportion of elastic energy $\left(W_{\mathrm{el}}\right)$ stored during the negative phase and released in the positive phase. $W_{\text {el }}$ was calculated by considering $E_{\text {meta }}$ as the sum of the metabolic energy associated to positive and negative work:

$E_{\text {meta }}=\left(W_{\text {pos }}-W_{\mathrm{el}}\right) \eta_{c c+}^{-1}+\left(W_{\text {neg }}-W_{\mathrm{el}}\right) \eta_{c c-}^{-1}$.

Since $W_{\text {pos }}=W_{\text {neg }}$ (see Table 1$)$ :

$W_{\mathrm{el}}(\mathbf{J})=W_{\text {pos }}-E_{\text {meta }}\left(\eta_{c c-} \times \eta_{c c+}\right)\left(\eta_{c c-}+\eta_{c c+}\right)^{-1}$

where $\eta_{c c}+$ and $\eta_{c c}$ - are the mechanical efficiencies of pure positive and negative work, which are 0.171 and 0.802 , respectively, for extensions and flexions of the lower limbs (Aura and Komi 1986). Inserting these values into Eq. 6 , since $\eta_{\text {net }}=W_{\text {ext }} E_{\text {meta }}^{-1}=\left(W_{\text {pos }}+W_{\text {neg }}\right)$ $E_{\text {meta }}^{-1}$ (see above) and rearranging one obtains:

$W_{\text {el }} \times W_{\text {pos }}^{-1}=1-0.282 \times \eta_{\text {net }}^{-1}$.

This equation allows one to calculate the recovery of elastic energy as a fraction of the positive work performed (see Fig. 2).
The metabolic energy associated with positive work $\left(E_{\mathrm{pos}}\right)$ was calculated using the following formula:

$E_{\text {pos }}(\mathbf{J})=E_{\text {meta }}-\left(W_{\text {pos }}-W_{\mathrm{el}}\right) \eta_{c c-}^{-1}$.

The mechanical efficiency of positive work $\left(\eta_{\text {pos }}\right)$ was calculated as the ratio of $W_{\text {pos }}$ to $E_{\text {pos }}$.

The relationship between $\eta_{\text {pos }}$ and $T$ was described by using an exponential model:

$\eta_{\mathrm{pos}}=a+b \mathrm{e}^{-T \cdot c^{-1}}$.

The three parameters of the exponential model $(a, b$ and $c$ in Eq. 8) were determined with an iterative procedure by minimising the sum of the mean squares of the differences between the estimated $\eta_{\text {pos }}$ and the measured $\eta_{\text {pos }}$ for the individual values of each subject (model 1) or the values of each series (model 2). This operation was done by a solver program. Figure 1 shows a mathematical property of the considered exponential model. When the value of $T$ is equivalent to $c, \eta_{\text {pos }}$ is equal to $a+36.79 \% \times b$. And a $T$ value of $4 c$ is associated with a $\eta_{\text {pos }}$ value of only $a+1.83 \% \times b$. The value of $T$ at this point $(4 c ; a+1.83 \% \times b)$ was considered to be the minimal $T$ required $\left(T_{\mathrm{lim}}\right)$ so that $\eta_{\text {pos }}$ reached its minimal value.

The minimal mechanical efficiency of positive work $\left(\eta_{\text {pos min }}\right)$ was calculated for each subject by setting $T$ equal to $4 c$ in the individual model (Eq. 8). Then, the $\eta_{\text {pos }}$ values for each of the four series were expressed as a percentage of $\eta_{\text {pos min }}\left(\% \eta_{\text {pos min }}\right)$. A second global model (model 3), estimating $\% \eta_{\text {pos min }}$ for all values of $T$, was calculated with the method described above:

$\% \eta_{\mathrm{pos} \min }=a+b \mathrm{e}^{-T \cdot c^{-1}}$.

Statistical analysis

All data are presented as mean (SD). The statistical analysis was performed with SPSS 13.0.1 (Chicago,
Fig. 1 Exponential equation used to model the relationship between $\eta_{\text {pos }}$ and $T$. A $T$ value of $4 c\left(T_{\mathrm{lim}}\right)$ is associated with a $\eta_{\text {pos }}$ value of only $a+1.83 \% \times b$

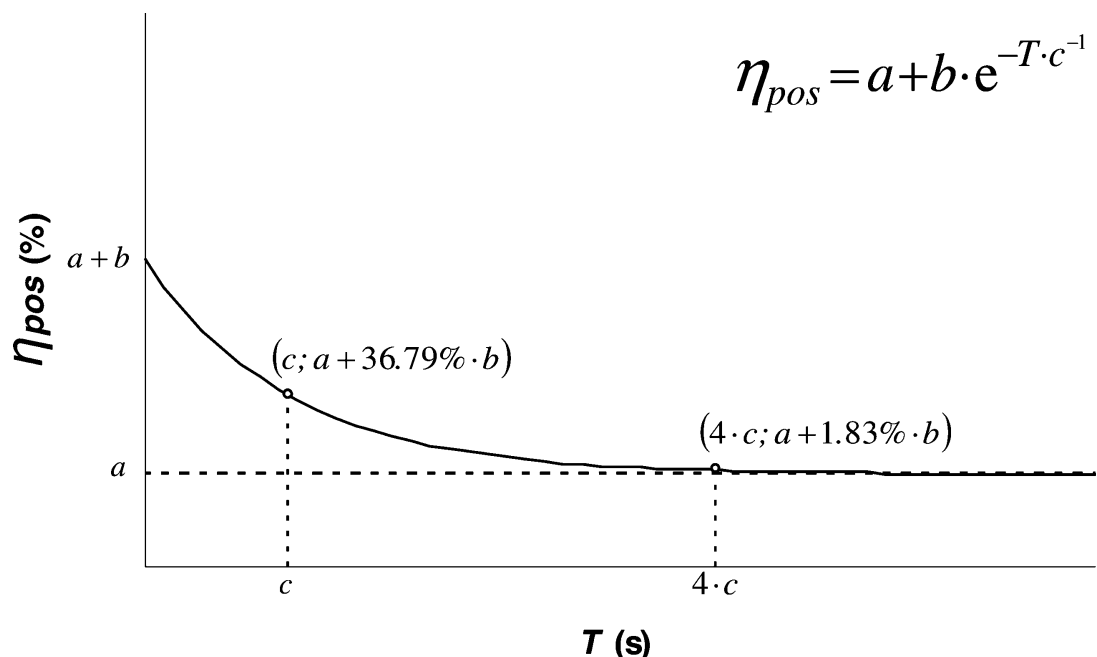


Table 1 Data [mean (SD)] measured in series $S_{0}, S_{1}, S_{2}$ and $S_{3}$ : transition time $(T)$, positive work $\left(W_{\text {pos }}\right)$, negative work $\left(W_{\text {neg }}\right)$, metabolic energy expenditure $\left(E_{\text {meta }}\right)$, net mechanical efficiency $\left(\eta_{\text {net }}\right)$, net mechanical efficiency of positive work $\left(\eta_{\text {pos }}\right)$, net mechanical efficiency of positive work expressed in percentage of its minimal value $\left(\% \eta_{\text {pos } \min }\right)$, flexion average speed $\left(v_{\mathrm{ff}}\right)$, extension average speed $\left(v_{\mathrm{ex}}\right)$ and vertical height difference between the squatting and standing-up positions $\left(h_{\mathrm{v}}\right)$

\begin{tabular}{|c|c|c|c|c|}
\hline & $S_{0}$ & $S_{1}$ & $S_{2}$ & $S_{3}$ \\
\hline$T(\mathrm{~s})$ & $0.02(0.01)^{*}, * *, * * *$ & $0.12(0.03)^{*, * *}$ & $0.24(0.09)^{*}$ & $0.56(0.08)$ \\
\hline$W_{\mathrm{pos}}(\mathrm{kJ})$ & $3.86(1.06)$ & $3.72(1.02)$ & $3.79(1.08)$ & $3.93(1.06)$ \\
\hline$W_{\text {neg }}(\mathrm{kJ})$ & $3.76(1.05)$ & $3.66(1.06)$ & $3.67(1.07)$ & $3.75(1.04)$ \\
\hline$E_{\text {meta }}(\mathrm{kJ})$ & $15.44(5.40)$ & $16.62(5.33)$ & $18.23(6.97)$ & $20.46(6.73)$ \\
\hline$\eta_{\text {net }}(\%)$ & $50.7(9.3)^{*}$ & $45.3(8.1)$ & $42.2(7.2)$ & $38.2(6.1)$ \\
\hline$\eta_{\text {pos }}(\%)$ & $31.1(5.5)^{*}$ & $27.7(4.7)$ & $26.0(4.4)$ & $23.8(3.8)$ \\
\hline$\% \eta_{\text {pos min }}(\%)$ & $131.6(8.4)^{*, * *, * * *}$ & $117.4(10.8)^{*}$ & $110.0(7.3)^{*}$ & $100.5(3.8)$ \\
\hline$v_{\mathrm{f}}\left(\mathrm{m} \mathrm{s}^{-1}\right)$ & $0.62(0.07)^{*, * *, * * *}$ & $0.51(0.07)$ & $0.48(0.05)$ & $0.48(0.08)$ \\
\hline$v_{\text {ex }}\left(\mathrm{m} \mathrm{s}^{-1}\right)$ & $0.65(0.04)^{*}, * *, * * *$ & $0.59(0.04)$ & $0.59(0.05)$ & $0.59(0.06)$ \\
\hline$h_{\mathrm{v}}(\mathrm{m})$ & $0.257(0.041)$ & $0.251(0.042)$ & $0.253(0.044)$ & $0.256(0.041$ \\
\hline
\end{tabular}

*Significant difference with series $S_{3}(P<0.05)$

${ }^{* *}$ Significant difference with series $S_{2}(P<0.05)$

${ }^{* * * *}$ Significant difference with series $S_{1}(P<0.05)$

USA). Since the distribution of the variables was not normal, a two-independent-samples test (Mann-Whitney) was used to identify differences in $T, W_{\text {pos }}, W_{\text {neg }}$, $E_{\text {meta }}, \eta_{\text {net }}, \eta_{\text {pos }}, \% \eta_{\text {pos min }}, W_{\text {el }}, v_{\text {fl }}, v_{\text {ex }}$ and $h_{\mathrm{v}}$ between the four series. Linear regressions were used to determine the degree of significance of the exponential models. The dependant variables were the measured values and the independent variables were the values estimated by the exponential models. For all tests, significance was declared when $P<0.05$.

\section{Results}

$T$ was significantly different between the four series (Table 1). As expected, there was no significant difference between $W_{\text {pos }}$ and $W_{\text {neg }}$ for the values of the four series altogether as well as between the four series (Table 1). $E_{\text {meta }}$ ranged between $15.44(5.40) \mathrm{kJ}$ (series $S_{0}$ ) and $20.46(6.73) \mathrm{kJ}$ (series $S_{3}$ ). The difference between these two series was, however, not significant $(P=0.064)$. The measured mechanical efficiencies were significantly higher in series $S_{0}$ than in series $S_{3}$
( $P=0.009$ and 0.013 for $\eta_{\text {net }}$ and $\eta_{\text {pos }}$, respectively). The efficiencies ranged between $38.2(6.1) \%$ (series $S_{3}$ ) and $50.7(9.3) \%$ (series $S_{0}$ ) for $\eta_{\text {net }}$ and $23.8(3.8) \%$ (series $S_{3}$ ) and $31.1(5.5) \%$ (series $S_{0}$ ) for $\eta_{\text {pos }}$ (Table 1). \% $\eta_{\text {pos min }}$ was significantly different between all series except $S_{1}$ vs $S_{2}(P=0.18$; Table 1$)$. The amount of negative work released as elastic energy $\left(W_{\mathrm{el}}\right)$, shown in Fig. 2, was significantly different between series $S_{0}$ and $S_{3}$ $(P=0.013)$. The results ranged between $26.5(11.5) \%$ (series $S_{3}$ ) and $43.7(9.2) \%$ (series $S_{0}$ ). Concerning the speed of execution, $v_{\mathrm{ff}}$ was higher in series $S_{0}$ than in series $S_{3}(P=0.006), S_{2}(P=0.002)$ and $S_{1}(P=0.018)$. Similarly, $v_{\text {ex }}$ was higher in series $S_{0}$ than in series $S_{3}$ $(P=0.035), S_{2}(P=0.035)$ and $S_{1}(P=0.035)$. No differences in $h_{\mathrm{v}}$ were observed between the four series (Table 1).

$\eta_{\text {pos }}$ was precisely predicted by model $1\left(R^{2}=0.975\right.$, $P<0.001)$ and, to a lesser extent, by model $2\left(R^{2}=0.342\right.$, $P=0.001)$. The mean value of $T_{\text {lim }}$, predicted by model 1 for each subject, was equal to $0.65 \mathrm{~s}$. The $T_{\lim }$ value, calculated using model 2 , was equal to $0.39 \mathrm{~s}$.

The relationship between $\% \eta_{\text {pos min }}$ and $T$ is plotted in Fig. 3. The resulting exponential equation of model 3
Fig. 2 Elastic energy release $\left(W_{\mathrm{el}}\right)$ during the positive phase in percentage of the work done during the negative phase for flexion-extensions of the lower limbs

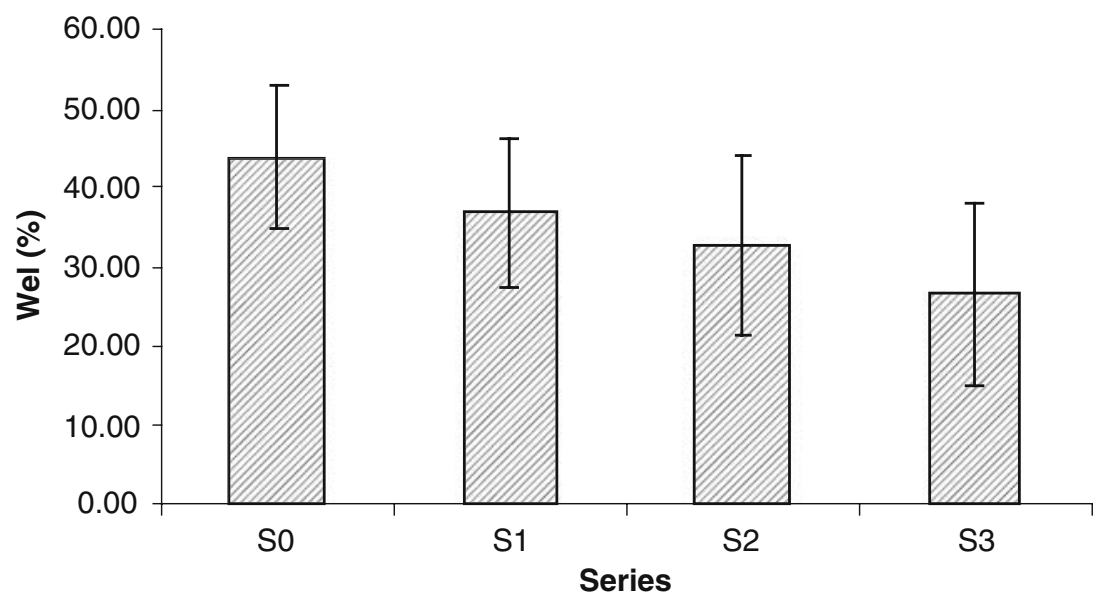


Fig. 3 Relationship between net mechanical efficiency of positive work $\left(\eta_{\text {pos }}\right)$ expressed in percentage of its minimal value $\left(\% \eta_{\text {pos min }}\right)$ and the transition time $(T)$, as calculated by model 3. The mean values and standard deviations of series $S_{0}$, $S_{1}, S_{2}$ and $S_{3}$ are indicated

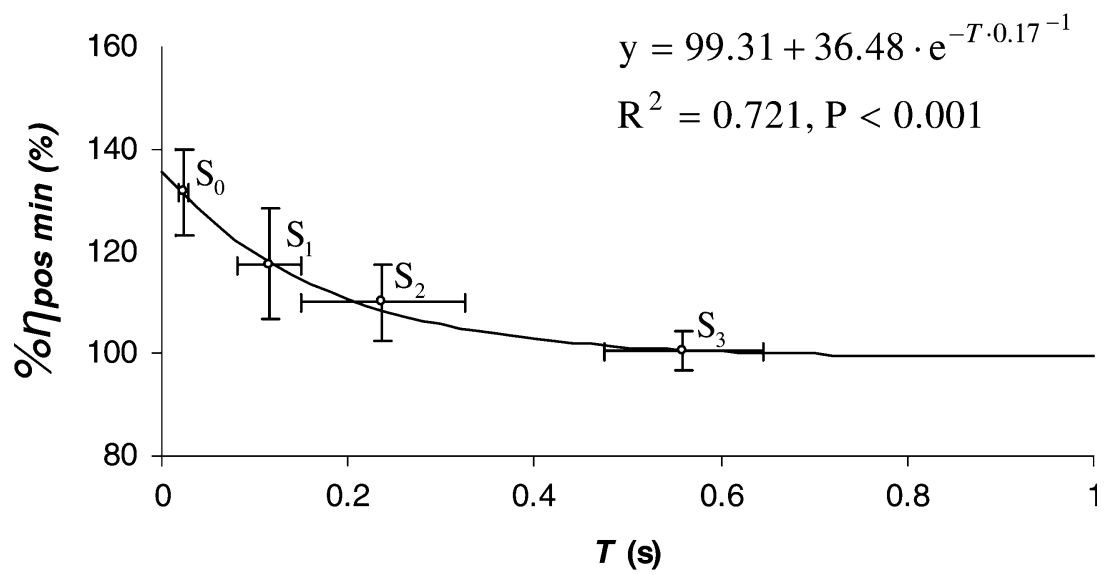
was $\% \eta_{\text {pos } \min }=99.31+36.48 \mathrm{e}^{-T \cdot 0.17^{-1}} \quad\left(R^{2}=0.721\right.$,
$P<0.001)$. The $T_{\lim }$ value, calculated using model 3 , was equal to $0.68 \mathrm{~s}$.

\section{Discussion}

The most important finding of this study is that mechanical efficiency decreased exponentially as the transition time between negative and positive work increased.

\section{Mechanical efficiency values}

The present study confirms results from previous studies that $\eta_{\text {net }}$ is significantly higher when the transition time is shorter. Thys et al. (1972) measured a mechanical efficiency of $25.8 \%$ in a rebound condition, comparable to series $S_{0}$ of the present study, and $18.8 \%$ in a norebound condition, comparable to series $S_{3}$. Since they disregarded the negative work and calculated the gross mechanical efficiency, their numerical findings are not directly comparable to the $\eta_{\text {net }}$ values of the present paper. Calculating mechanical efficiencies for the current study with the method used by Thys et al. gives similar results (25.7 and $19.6 \%$ for series $S_{0}$ and $S_{3}$, respectively). Moreover, the transition time between flexions and extensions is almost the same in the two studies. Thys et al. reported a transition time of $0.6 \mathrm{~s}$ for their no-rebound condition and $T$ has been measured to be $0.56 \mathrm{~s}$ in series $S_{3}$ of the present study.

Maximum $\eta_{\text {net }}$ values of $\sim 25 \%$ (Gibbs and Chapman 1974) have been measured under in vitro conditions. The greater $\eta_{\text {net }}$ values in the present study are due to a low energetic cost of the negative work performed during the flexion, and a storage and re-use of elastic energy. Since the present paper focuses on the influence of the transition time on the mechanical efficiency, the influence of this factor was isolated in the calculation of $\eta_{\text {pos }}$. Its value for series $S_{3}(23.8 \%)$ is comparable to the $25 \%$ efficiency of isolated muscle or to the net mechanical efficiency associated with pure concentric activities, such as cycling, which was assessed to $24 \%$ (Gaesser and Brooks 1975).

\section{Elastic energy restitution}

Series $S_{3}$ was associated to a $\% \eta_{\text {pos min }}$ value of 100.5 (3.8) $\%$, indicating that the minimal value of $\eta_{\text {pos }}$ is reached for a $T$ value close to that of series $S_{3}$. $W_{\mathrm{el}}$ was not nil for series $S_{3}$ but equal to $26.5(11.5) \%$. Therefore, there is still an elastic energy restitution when $\eta_{\text {pos }}$ has reached its minimal value. This is probably due to the fact that the extensor muscles of the lower limbs remained contracted during the squatting posture held by the subjects. If the muscles had been allowed to relax, the $26.5 \% W_{\text {el }}$ of series $S_{3}$ would probably have turned into heat, as Thys et al. (1972) mentioned.

The elastic energy restitution value of $43.7 \%$ obtained for series $S_{0}$ is lower than the results of Bosco et al. (1997) who calculated an elastic energy restitution of $50 \%$ for vertical jumps. This small difference is normal because jumping involves much higher force values than the flexion-extension manoeuvre in the present paper. The related stretch and shortening of elastic structures is certainly larger and consequently the amount of elastic energy stored and released is more important.

\section{Relationship between $\eta_{\text {pos }}$ and $T$}

The exponential equation used to model $\eta_{\text {pos }}$ according to $T$ is adequate if it is individually applied (model $1, R^{2}$ $=0.975$ ). The global model, in the other hand, is not a good predictor of $\eta_{\text {pos }}$ (model 2, $R^{2}=0.342$ ). The major difficulty in modelling $\eta_{\text {pos }}$ is the heterogeneity of the individual values for a given $T$ value. This assumption is confirmed by the higher goodness-of-fit of model 3 
$\left(R^{2}=0.746\right)$, which has the property to set the asymptote of the exponential curve to the constant value of $100 \%$.

Subjects who are very efficient at low transition time values compared to the other subjects are also very efficient at high transition time values. These interpersonal differences may be explained by the repartition of muscle fibre types. Efficiency in slow muscles was found to be greater than in fast muscles (Aura and Komi 1987; Gibbs and Gibson 1972; Wendt and Gibbs 1973). Muscle active stiffness was also mentioned as affecting mechanical efficiency. It has been observed that the energy cost of running was negatively related to muscle active stiffness (Dalleau et al. 1998). However, this second factor is a consequence of the first. Actually, after an endurance-training period, the rat soleus muscle changes were an increase of active stiffness associated with an increase of slow muscle fibres. The opposite change was found as a result of strength training (Goubel and Marini 1987; Pousson et al. 1991). Therefore, a higher percentage of type I fibres, and consequently higher muscle active stiffness, might explain the highest values of mechanical efficiencies among the seven subjects of the present study. This hypothesis could be corroborated by subsequent studies.

Since model 2 is imprecise due to the heterogeneity of individual $\eta_{\text {pos }}$ values at a given $T$ value, it can be hypothesized that this model could be improved by accounting for individual $\eta_{\text {pos min }}$ values. One way to verify this is to estimate $\eta_{\text {pos }}$ using model 2 (Eq. 8) but with substituting the $a$ parameter for $\eta_{\text {pos min }}$ (model 4). The resulting exponential equation is $\eta_{\text {pos }}=\eta_{\text {pos } \min ^{+}}$ $8.71 \mathrm{e}^{-T \cdot 0.15^{-1}}\left(R^{2}=0.915, P<0.001\right)$ and $T_{\lim }$ is calculated to be $0.59 \mathrm{~s}$. Model 4 , taking into account the heterogeneity of individual $\eta_{\text {pos }}$ values at a given $T$ value, and being a better predictor of $\eta_{\text {pos }}$ than model 3 , will be considered as the reference in the calculation of $T_{\mathrm{lim}}$.

\section{Protocol}

The validity of the experimental protocol can be assessed by the temporal data. The expected values of $T(0.25,0.5$ and $0.75 \mathrm{~s}$ for series $S_{1}, S_{2}$ and $S_{3}$, respectively) are greater than the measured values $(0.12,0.24$ and $0.56 \mathrm{~s}$, respectively), suggesting that the subjects delayed the beginning of the flexion phase or anticipated the beginning of the extension phase. This point is of little pertinence because the timer was used with the aim of varying $T$. Since this parameter was significantly different between all series, the subjects executed the rhythms correctly.

The vertical displacement of the centre of body mass $\left(h_{\mathrm{v}}\right)$ was essentially the same between all four series. Therefore, the stretching amplitude of the extensor muscles was constant. The verification of this part of the experimental protocol is critical because the stretching amplitude was identified as affecting the elastic energy recoil (Bosco et al. 1982).
The different $v_{\text {ex }}$ observed between series $S_{0}$ and the three others was not expected. In a general sense, the force/velocity diagram of muscles would imply that mechanical efficiency was affected by the contraction speed. However, it has been shown that the mechanical efficiency of positive work is not correlated with the shortening speed when the contraction speed varies with a narrow range of angular velocities (Komi et al. 1987), as it is the case in the present study. Therefore, the heterogeneity detected in the extension speed did not affect the results. There was also an unexpected difference between series $S_{0}$ and the three others concerning $v_{\mathrm{fl}}$. This probably affected the results because the possibility for the muscle to store and reuse elastic energy is improved if the stretching speed is high (Bosco et al. 1981). Moreover, the mechanical efficiency of negative work was found to increase with increased stretch velocity (Komi et al. 1987). Therefore, the relationship between $\eta_{\text {pos }}$ and $T$ may have been influenced by the flexion speed. The present study has even so the merit to have measured this parameter, that is difficult to control under in vivo conditions.

The assumption of the pure negative and positive efficiencies as 80.2 and $17.1 \%$ may be criticized. Aura and Komi (1986) stated that $\eta_{c c}+$ and $\eta_{c c-}$ should be measured individually for each subject in cases where they are used for calculating the mechanical efficiency of positive work in SSC situations. However, they estimated the numerical values of $\eta_{c c-}$ in different locomotive activities including flexion-extension of the lower limbs for which $\eta_{c c}$ - ranged between 70 and $90 \%$. Assuming a $\eta_{c c-}$ value of 70 or $90 \%$ and a $\eta_{c c}+$ value of 15 or $20 \%$ in the present study leads to a $W_{\text {el }}$ value change of only $7.7(2.0) \%$ and to a $\eta_{\text {pos }}$ value change of only $1.8(2.5) \%$.

Concerning the Kistler force platform, it may be questioned whether the length of the series (4 min) was too long to exclude that some drift of the force signal was generated by the piezoelectric crystals. Actually the drift was calculated for each subject and was equal to $0.74(0.47) \mathrm{N} \mathrm{min}{ }^{-1}$ which represents only $0.11(0.07) \%$ error.

\section{Conclusion}

The transition time between flexions and extensions of the lower limbs was clearly identified as a key parameter affecting the mechanical efficiency associated with the exercise. Overall net mechanical efficiency of positive work as well as net mechanical efficiency decreased as the transition time increased. The relationship between mechanical efficiency of positive work and the transition time was expressed by exponential equations. The minimal transition time required so that the net mechanical efficiency of positive work reached its minimal value was $0.59 \mathrm{~s}$. 
Acknowledgements The experiments comply with current Swiss laws.

\section{References}

Asmussen E, Bonde-Petersen F (1974) Apparent efficiency and storage of elastic energy in human muscles during exercise. Acta Physiol Scand 92:537-545

Aura O, Komi PV (1986) Mechanical efficiency of pure positive and pure negative work with special reference to the work intensity. Int J Sports Med 7:44-49

Aura O, Komi PV (1987) Effects of muscle fiber distribution on the mechanical efficiency of human locomotion. Int J Sports Med 8(Suppl 1):30-37

Bosco C, Komi PV, Ito A (1981) Prestretch potentiation of human skeletal muscle during ballistic movement. Acta Physiol Scand 111:135-140

Bosco C, Ito A, Komi PV, Luhtanen P, Rahkila P, Rusko H, Viitasalo JT (1982) Neuromuscular function and mechanical efficiency of human leg extensor muscles during jumping exercises. Acta Physiol Scand 114:543-550

Bosco C, Saggini R, Viru A (1997) The influence of different floor stiffness on mechanical efficiency of leg extensor muscle. Ergonomics 40:670-679

Cavagna GA, Saibene FP, Margaria R (1964) Mechanical work in running. J Appl Physiol 19:249-256

Cavagna GA, Mazzanti M, Heglund NC, Citterio G (1985) Storage and release of mechanical energy by active muscle: a non-elastic mechanism? J Exp Biol 115:79-87

Dalleau G, Belli A, Bourdin M, Lacour JR (1998) The spring-mass model and the energy cost of treadmill running. Eur $\mathbf{J}$ Appl Physiol Occup Physiol 77:257-263
Gaesser GA, Brooks GA (1975) Muscular efficiency during steadyrate exercise: effects of speed and work rate. J Appl Physiol 38:1132-1139

Gibbs CL, Chapman JB (1974) Effects of stimulus conditions, temperature, and length on energy output of frog and toad sartorius. Am J Physiol 227:964-971

Gibbs CL, Gibson WR (1972) Energy production of rat soleus muscle. Am J Physiol 223:864-871

Goubel F, Marini JF (1987) Fibre type transition and stiffness modification of soleus muscle of trained rats. Pflugers Arch 410:321-325

Komi PV, Kaneko M, Aura O (1987) EMG activity of the leg extensor muscles with special reference to mechanical efficiency in concentric and eccentric exercise. Int J Sports Med 8(Suppl 1):22-29

Pousson M, Perot C, Goubel F (1991) Stiffness changes and fibre type transitions in rat soleus muscle produced by jumping training. Pflugers Arch 419:127-130

Stainbsy WN, Gladden LB, Barclay JK, Wilson BA (1980) Exercise efficiency: validity of base-line subtractions. J Appl Physiol 48:518-522

Thys H, Faraggiana T, Margaria R (1972) Utilization of muscle elasticity in exercise. J Appl Physiol 32:491-494

Thys H, Cavagna GA, Margaria R (1975) The role played by elasticity in an exercise involving movements of small amplitude. Pflugers Arch 354:281-286

Wendt IR, Gibbs CL (1973) Energy production of rat extensor digitorum longus muscle. Am J Physiol 224:1081-1086

Whipp BJ, Wasserman K (1972) Oxygen uptake kinetics for various intensities of constant-load work. J Appl Physiol 33:351356 